\title{
Back beliefs among elderly seeking health care due to back pain; psychometric properties of the Norwegian version of the back beliefs questionnaire
}

Alexander Tingulstad ${ }^{1 *}$ (D), Rikke Munk ${ }^{1}$, Margreth Grotle ${ }^{1,2}$, Ørjan Vigdal $^{1}$, Kjersti Storheim $^{1,2}$ and Birgitta Langhammer ${ }^{1,3}$

\begin{abstract}
Background: The Back Beliefs Questionnaire (BBQ) is a 14-item patient-reported questionnaire that measures attitudes and beliefs about the consequences of back pain. The BBQ has recently been translated into Norwegian, but its psychometric properties have not yet been tested. The aim of this study is to evaluate the reliability and construct validity of the BBQ when used on elderly patients with back pain.

Method: A prospective cohort study with a test-retest design among 116 elderly patients ( $\geq 55$ years of age) seeking primary care for a new episode of back pain. Test-retest, standard error of measurement (SEM), minimal detectable change (MDC), internal consistency and construct validity by a priori hypotheses (Spearman's- and Pearson correlation coefficient) were tested.

Results: A total of 116 patients, mean age (SD) 67.7 (8.3), were included and 63 patients responded to the testretest assessment. The mean (SD) BBQ sum scores (range 9-45) were 29.8 (7.0) and 29.2 (6.7) for the test and retest respectively. The test-retest was acceptable with an intraclass correlation coefficient of $0.71(95 \% \mathrm{Cl}, 0.54-0.82)$, SEM was 3.8 and MDC 10.5. Internal consistency with Cronbach's alpha was good (0.82) and acceptable construct validity was supported by the confirmation of $75 \%$ of the a priori hypotheses.
\end{abstract}

Conclusion: The Norwegian version of the $\mathrm{BBQ}$ demonstrated acceptable test-retest reliability and good construct validity and can be used to assess pessimistic beliefs in elderly patients with back pain.

Keywords: Back beliefs questionnaire, BBQ, Back pain, Validity, Reliability, Elderly

\section{Introduction}

Back pain is among the most common musculoskeletal complaints seen in primary care [1]. The prevalence of back pain has been rising continuously for many years and the financial burden on society is increasing [1-3]. Although our population is aging globally, the elderly are often excluded from research on back pain and the influence of psychological factors [4]. Psychological factors, in particular beliefs about back pain, have been shown to play a major role for the course of back pain

\footnotetext{
* Correspondence: alting@oslomet.no

Oslo Metropolitan University, Pilestredet 44, 0167 Oslo, Norway

Full list of author information is available at the end of the article
}

[5-8]. Negative and irrational beliefs are associated with persistent back pain [5].

Beliefs and attitudes towards back pain can be measured with questionnaires. The Back Beliefs Questionnaire (BBQ) was developed by Symonds et al., with the aim to make a new instrument to measure beliefs and attitudes related to back pain [5]. The authors developed a 14-item self-report questionnaire to investigate beliefs about the inevitable consequences of back pain [5]. BBQ has been used to predict recovery rate from back pain $[9,10]$, in population studies assessing public attitudes and as an outcome to assess effectiveness of educational campaigns [11-13]. To our knowledge, the BBQ has been translated into Arabic [14, 15], German [10], Chinese [16, 17] and

(c) The Author(s). 2019 Open Access This article is distributed under the terms of the Creative Commons Attribution 4.0 International License (http://creativecommons.org/licenses/by/4.0/), which permits unrestricted use, distribution, and reproduction in any medium, provided you give appropriate credit to the original author(s) and the source, provide a link to the Creative Commons license, and indicate if changes were made. The Creative Commons Public Domain Dedication waiver (http://creativecommons.org/publicdomain/zero/1.0/) applies to the data made available in this article, unless otherwise stated. 
French [18]. Most of these methodological studies have shown good test-retest reliability and validity. However, if BBQ is to be used as a measurement outcome in addition to a predictor, test-retest reliability in terms of minimal detectable change (MDC), standard error of measurement (SEM) and limits of agreement (LOA) needs to be established. These estimates are useful as they provide an interpretation of measurement error according to the absolute score of an instrument [19], however, few studies have investigated these properties $[14,15]$.

The BBQ has recently been translated into Norwegian, but assessment of test-retest reliability and validity has not yet been performed. The psychometric properties of any scale may be affected by translation into another language, hence, it is important for the scale to be evaluated psychometrically. Therefore, the aim of this study was to evaluate the psychometric properties of the Norwegian version of the BBQ in terms of test-retest reliability, construct validity and internal consistency when used on elderly patients with a new episode of back pain.

\section{Material and methods}

This methodological study is part of the BACE (BACk Complaints in Elders) study in Norway. BACE is a prospective cohort study designed to assess elderly $(\geq 55$ years) patients with back pain. The protocol has been published [20].

\section{Translation and cross-cultural adaption}

The English version of the $\mathrm{BBQ}$ was translated and cross-culturally adapted into Norwegian according to guidelines [21]. Two translators (one philologist and one clinician), whose mother tongue is Norwegian, independently translated the BBQ into Norwegian, and synthesized them into one Norwegian version. Two native English speakers, blinded to the original $\mathrm{BBQ}$, independently performed the back translation and synthesized the two versions into one English version. An expert committee consisting of the translators and two researchers from our research group (MG and RM) reviewed the translations and agreed on a prefinal version. The prefinal version was tested on ten participants at baseline with similar characteristics as the whole sample. The items were confirmed to be relevant and understandable without any proposed alterations. Hence, the final version of the Norwegian BBQ evaluated in this study is the same as the prefinal version.

\section{Participants}

Eligible participants were patients aged 55 years or older who had attended a consultation with a primary care practitioner regarding a new episode of back pain. Back pain was defined as pain from the cranial ridge of the scapula to S1. Patients were excluded if they had difficulty with completing the questionnaire due to language barriers or if they had received treatment for the same episode of back pain within the last 6 months. All patients received written and oral information about the study and informed consent was signed by all participants.

\section{Procedures and measurements}

The BBQ was administered to all participants as part of a comprehensive questionnaire used in the cohort. This included sociodemographic variables (age, gender, education, work status), medical history and several questionnaires [20]. The questionnaires were selfadministered by all patients, using a tablet computer, alone in a separate room. Baseline (T0) also consisted of a clinical examination of the patients [20]. Test-retest reliability was assessed by testing the BBQ on a subsample of the patients. At baseline, patients were asked to fill out the retest (T1) at home, until a sufficient number (>50) of participants had completed the retest. The participants were asked to fill out the second questionnaire 2 days after the baseline testing.

The $\mathrm{BBQ}$ is a patient-reported questionnaire consisting of 14 statements regarding beliefs about the consequences of back pain, with items such as "Back trouble will eventually stop you from working" and "Back trouble makes everything in life worse". Each item is scored on a 5-point Likert scale ranging from strongly disagree (1) to strongly agree (5). Five of the items are distractors and the remaining 9 items are used in the scoring of the questionnaire, resulting in a score ranging from 9 to 45. The scores are reversed before they are summarized, meaning that a low score indicates more pessimistic beliefs regarding the consequences of back pain [5]. The BBQ instrument is shown in the Additional file 1.

Several reference scales were used to evaluate convergent and divergent construct validity. For convergent validity we used, the Fear-Avoidance Beliefs Questionnaire for physical activity (FABQ-PA) [6], the Roland-Morris Disability Questionnaire (RMDQ) [22] and the Pain Catastrophizing Scale (PCS) [23], for divergent validity we used the Numeric Rating Scale (NRS). FABQ-PA consists of 5 statements that evaluates fear and avoidance behavior introduced through physical activity [6]. The questionnaire has been translated into Norwegian and has shown acceptable psychometric properties in Norwegian patients with low back pain [24]. Acceptable results were also obtained when assessing the reliability and validity of a Norwegian version of the RMDQ [25]. This questionnaire examines functional status related to normal activities of daily life [22]. PCS includes 13 items that focus on thoughts and feelings about pain [23]. A Norwegian 
version tested on patients with back pain has demonstrated acceptable psychometric properties [26]. NRS has been widely used to evaluate pain and has proven to be preferable when examining low back pain patients [27].

\section{Statistical analysis/analysis}

IBM SPSS Statistics for Windows, version 23.0 (IBM Corp, Armonk, NY) was used for all data analyses. Descriptive analyses included means and standard deviation (SD) for numerical variables or frequencies for categorical variables. The sample size was based on the quality criteria proposed by Terwee et al. [19]. This suggested criteria recommends a minimum of 50 participants when exploring test-retest reliability, and at least 100 participants when exploring internal consistency and construct validity. Kolmogorov-Smirnov tests for normality and the visual inspection of distribution plots were used to determine the distribution of the BBQ-scores.

Floor and ceiling effects were assessed by evaluating the numbers of participants with the lowest or highest score. They were considered to be present if more than $15 \%$ of the participants had the lowest or the highest possible score [19]. In addition, data quality was assessed by evaluating missing data.

Test-retest reliability was assessed by calculating the intraclass correlation coefficient (ICC) from a two-way random effects model, absolute agreement $(2,1)$. Since each subject was only measured once at the test and the retest, the single measure value is used in the results. A minimum ICC of 0.7 was considered acceptable [19]. Measurement error was demonstrated by the standard error of measurement (SEM) and minimal detectable change (MDC). The formula for SEM is SEM $=\mathrm{SD} \sqrt{ } 1-$ ICC and MDC are calculated with MDC $=1.96 \times \sqrt{ } 2 \times$ SEM. Bland-Altman plots were constructed to assess how much the scores can vary in stable persons throughout the scale, with the agreement of the testretest of the $\mathrm{BBQ}$. Limits of agreement (95\%) were calculated with the formula [mean difference $\pm 1.96 \times \mathrm{SD}_{\text {dif- }}$ ference] [28].

The internal consistency of the questionnaire was assessed with Cronbach's Alpha. Values ranging from 0 to 1 are considered good when above 0.8 , moderate between 0.7 and 0.8, and low when under 0.7 [29].

Construct validity for the BBQ total score was assessed by comparing the $\mathrm{BBQ}$ for association to concurrent measures. Predefined hypotheses of association were established based on the construct of the measures and former correlations in similar studies. The BBQ was hypothesized to have moderate to high correlation with the FABQ-PA, moderate correlation with the RMDQ, high correlation with the PCS, and low to moderate correlation with the NRS. A high score in reference scales indicates more fear avoidance, catastrophizing, disability and pain, and a low score in BBQ reflects pessimistic beliefs and attitudes, meaning that all correlations were expected to have negative values. The Pearson's correlation coefficient was used if values were normally distributed and the Spearman's correlation coefficient if values were not normally distributed. Correlation between scales was interpreted as high when $\mathrm{r}$ was 0.50 and above, moderate when between 0.30 and 0.49 , and low when between 0.10 and 0.29 [30]. An acceptable construct validity of the BBQ was obtained if at least $75 \%$ of the hypotheses were confirmed [19].

\section{Results}

\section{Patients and data quality}

The prefinal version of the Norwegian BBQ was tested on the first ten participants at baseline, with similar characteristics as the whole sample. The ten participants included 6 women and 4 men, with a mean age of 69.1, all with a history of back pain and a mean (SD) BBQ score of 27.6 (4.9). The main study included 116 patients from primary care with back pain in the validation (T0), 71 women and 45 men. Of those included, 10 patients had missing items in the $\mathrm{BBQ}$ and were excluded from the analysis, resulting in a total of 106 participants with valid data. Sixty-three participants had valid data at retest (T1). The 10 incomplete responses had a total of 25 missing items, within which every item on the scale was represented. Items 13 ("Back trouble must be rested") and 14 ("Later in life back trouble gets progressively worse") had five missing values each, and the other items each had 1-3 missing values. Among the distractors, item 7 had four missing values, and item 4,5,9 and 11 had $1-2$ missing values. The mean (SD) BBQ total score at T0 was 29.8 (7.03). The lowest (9) and highest (45) possible scores were each achieved by one participant, indicating no floor- or ceiling effects for the BBQ total score. Patient characteristics and clinical variables are presented in Table 1.

\section{Reproducibility}

The median time between test and retest was 3 days (range 1-13). There was an acceptable agreement between the test and retest total score, with an $\operatorname{ICC}(2,1)$ of 0.71 (95\% CI, 0.54-0.82). The SEM of the BBQ total score was 3.8, the MDC was 10.5 and MDC\% was 23.3. The Bland-Altman plot (Fig. 1) demonstrates a mean difference between test and retest of 1.6 points, and limits of agreement of 10.9 and -7.7 points of the total score from 9 to 45 . The internal consistency, assessed by Cronbach's alpha, was 0.82 for the BBQ at T0. At T1, BBQ demonstrated a Cronbach's alpha value of 0.80 .

\section{Construct validity}

Evaluation of normality with Kolmogorov-Smirnov tests and the visual inspection of distribution plots 
Table 1 Characteristics of patients in the whole sample (T0) $(n=116)$ and the test-retest sub-group (T1) $(n=63)$

\begin{tabular}{|c|c|c|c|c|}
\hline Age (yrs.) & 116 & $67.7(8.32)$ & 63 & $68.6(8.69)$ \\
\hline Sex (\% Women) & 116 & $71(61)$ & 63 & $38(60)$ \\
\hline Civil status (\%) & 115 & & 62 & \\
\hline Married/cohabiting & & $85(73)$ & & $47(76)$ \\
\hline Single & & $19(16)$ & & $8(13)$ \\
\hline Widow/widower & & $11(10)$ & & $7(11)$ \\
\hline Language (\%) & 114 & & 61 & \\
\hline Norwegian & & $109(94)$ & & $59(97)$ \\
\hline Other & & $5(4)$ & & $2(3)$ \\
\hline Education (< 12 years) (\%) & 113 & $62(53)$ & 61 & $29(48)$ \\
\hline Pain medication (yes) (\%) & 115 & $50(43)$ & 62 & $25(40)$ \\
\hline Employed (\%) & 95 & $35(37)$ & 48 & $17(35)$ \\
\hline Smoking (yes) (\%) & 115 & $10(9)$ & 62 & $3(5)$ \\
\hline Expectations about back pain in 3 months (\%) & 114 & & 63 & \\
\hline Healed & & $21(18)$ & & $12(19)$ \\
\hline Much better & & $68(59)$ & & $39(62)$ \\
\hline No change & & $24(21)$ & & $11(18)$ \\
\hline Much worse & & $1(1)$ & & $1(2)$ \\
\hline Worse than ever & & $0(0)$ & & $0(0)$ \\
\hline History of back pain (yes) (\%) & 114 & $111(97)$ & 62 & $61(98)$ \\
\hline Frequency of back pain (\%) & 109 & & 60 & \\
\hline Monthly & & $42(39)$ & & $24(40)$ \\
\hline Yearly & & $36(33)$ & & $21(35)$ \\
\hline Less than once per year & & $19(17)$ & & $10(17)$ \\
\hline Once every 5 years & & $9(8)$ & & $4(7)$ \\
\hline One time & & $3(3)$ & & $1(2)$ \\
\hline BBQ (9-45), mean (SD) & 106 & $29.8(7.03)$ & 57 & $29.2(6.74)$ \\
\hline NRS (0-10), median (range) & 110 & $6(0-10)$ & 61 & $5.4(0-10)$ \\
\hline FABQ-PA (0-24), mean (SD) & 116 & $10.1(6.08)$ & 63 & $10.1(6.78)$ \\
\hline PCS (0-52), median (range) & 116 & $12(0-48)$ & 63 & $11.8(0-39)$ \\
\hline RMDQ (0-24). mean (SD) & 116 & $10(5.13)$ & 63 & $9.8(4.79)$ \\
\hline
\end{tabular}

BBQ Back Beliefs Questionnaire; NRS Numeric Rating Scale; FABQ Fear-Avoidance Beliefs Questionnaire; RMDQ Roland-Morris Disability Questionnaire; PCS Pain Catastrophizing Scale

suggested that BBQ, FABQ-PA and RMDQ, were normally distributed, while PCS and NRS were nonnormally distributed. Table 2 presents the predefined hypotheses and the correlation analyses between $\mathrm{BBQ}$ and FABQ-PA, PCS, RMDQ and NRS. Reversal of the $\mathrm{BBQ}$ score leads to negative correlation coefficients. The BBQ total score showed a strong correlation coefficient to the FABQ-PA $(r=-0.57)$ and, moderate correlation to both PCS (rho $=-0.45)$ and RMDQ $(r=-0.49)$. Moreover, the BBQ demonstrated a weak correlation to the NRS (rho $=-0.14)$. In total, the correlation coefficients confirmed $75 \%$ of the a priori hypotheses.

\section{Discussion}

The Norwegian version of the BBQ shows acceptable psychometric properties in elderly patients with a new episode of back pain. Our results indicate that BBQ can be used in both clinical settings and research with the purpose of assessing beliefs about back pain. This is in line with former assessments of BBQ in other languages $[14,15,31]$. Our study is the first report to evaluate psychometric properties in the Norwegian version of the $\mathrm{BBQ}$. Additionally, the fact that our research was conducted in elderly patients with back pain contributes important knowledge to a field in which most research has been conducted in younger populations [4, 32]. 


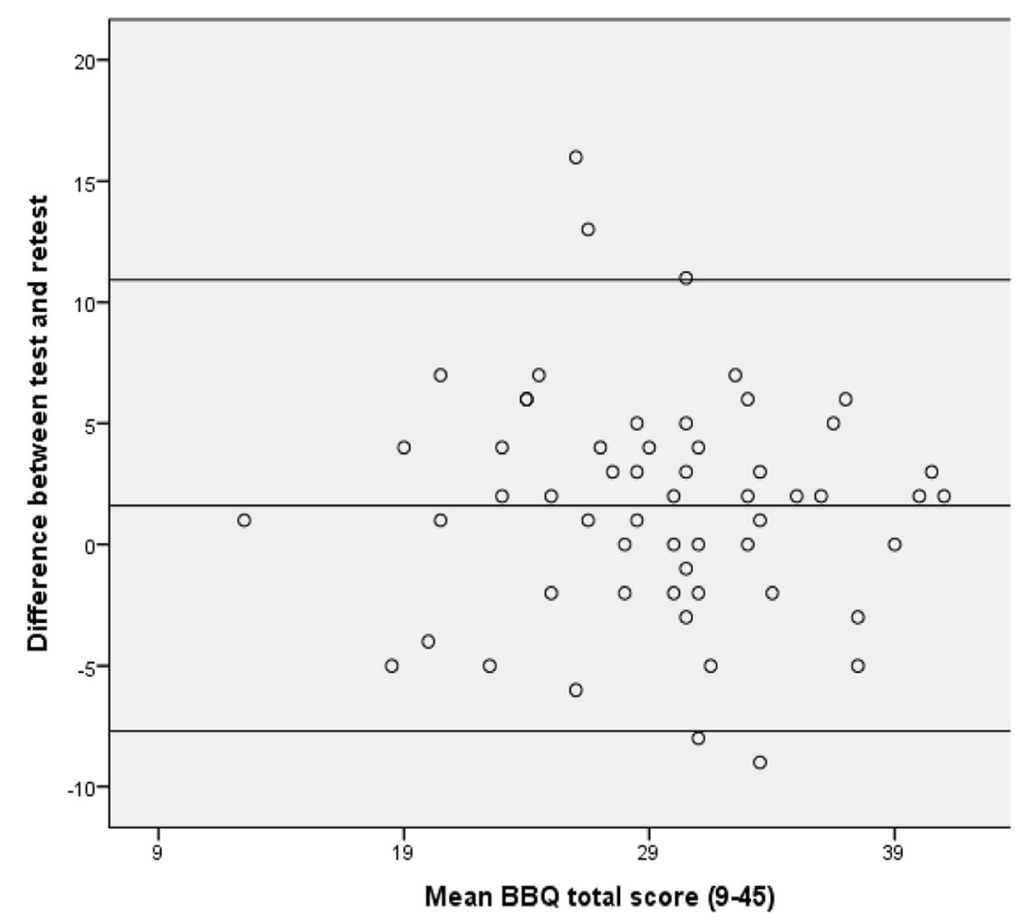

Fig. 1 Bland-Altman plot of mean difference between test and retest of the Back Beliefs Questionnaire $(n=57)$ and the Limits of Agreement

Fears and beliefs leading to avoidance have been shown to negatively influence the prognosis of back pain and increases the risk of developing chronic disability [9, 33]. With $B B Q$ as an examination measurement, it is possible to detect negative beliefs in patients with back pain. Early detection will allow primary healthcare workers to provide back pain patients with clarifying information pertaining to their irrational beliefs. This positive influence may have an important socioeconomic impact worldwide.

Our study sample completed the BBQ with a mean score of approximately 30 on a scale ranging from 9 to 45 ; this is a relatively high score, reflecting optimistic beliefs. This score is higher than some other studies which report a mean score ranging from 21 to $26[14,15,18$, $34]$, but similar to a study from Australia which reports a mean BBQ score of 30.7 [33]. The low level of negative beliefs in our sample of elderly people might have been influenced by different coping strategies, reduced pain perception and it might be argued that some elderly patients believe pain to be a normal part of the ageing process and have more realistic beliefs [35, 36]. Only $35 \%$ of our participants were working, and one could speculate that retired participants may experience fewer consequences due to an episode of back pain as they are unconcerned by the responsibilities of employment and with taking sick leave. Since previous studies have been conducted on different populations, such as healthcare workers [17], healthcare students [16], healthy individuals [11], and younger patients [18] as well as in different cultures [14, 15, 34], it is difficult to make any direct comparisons with this study. Furthermore, back beliefs

Table 2 Construct validity: a priori formulated hypothesis

\begin{tabular}{|c|c|c|c|}
\hline Hypothesis & Correlation value & $\mathrm{N}$ & $\begin{array}{l}\text { Hypothesis } \\
\text { confirmed? }\end{array}$ \\
\hline $\begin{array}{l}\text { The } \mathrm{BBQ} \text { was expected to have moderate to high } \\
\text { correlation with FABQ-PA }\end{array}$ & $-.573^{\mathrm{a}}$ & 106 & Yes \\
\hline $\begin{array}{l}\text { The BBQ was expected to have moderate } \\
\text { correlation with RMDQ }\end{array}$ & $-.494^{\mathrm{a}}$ & 106 & Yes \\
\hline $\begin{array}{l}\text { The BBQ was expected to have high correlation } \\
\text { with PCS }\end{array}$ & $-.447^{\mathrm{a}}$ & 106 & No \\
\hline $\begin{array}{l}\text { The BBQ was expected to have low to moderate } \\
\text { correlation with NRS }\end{array}$ & $-.138^{\mathrm{a}}$ & 101 & Yes \\
\hline
\end{tabular}

a Significant correlation (<0.01). BBQ Back Beliefs Questionnaire; NRS Numeric Rating Scale; FABQ Fear-Avoidance Beliefs Questionnaire; RMDQ Roland-Morris Disability Questionnaire; PCS Pain Catastrophizing Scale 
can change rapidly, which can influence evaluations using the $\mathrm{BBQ}$. While we found a low correlation between the BBQ and pain, in their research, Bostick and associates found that $\mathrm{BBQ}$ participants achieved lower scores when experiencing acute and severe pain, while a 1-week history of mild back pain resulted in higher scores [31]. The ICC was considered to be acceptable (0.71) according to our chosen classification, which suggests that the $\mathrm{BBQ}$ is a reliable outcome measure in our population [19]. In earlier studies, the BBQ has demonstrated ICC with results ranging from $0.80-0.89$ [10, 1417]. Few authors specify the chosen effects model and measure regarding the ICC, which can influence the outcome results. Measuring back beliefs with retest might be challenging. There is a potential risk of recall bias if the participant has a short interval between the test and the retest, and a risk of possible change in back pain status when the time between tests is long. A short interval was chosen in this study, since the high number of questionnaires completed at $\mathrm{T} 0$ would most likely reduce recall bias of $\mathrm{BBQ}$ at T1 2 days later. The ICC is influenced by the variation between the patients - heterogeneity resulting in a high ICC value - and substantiates the importance of assessing measurement errors [37]. MDC, which expresses an error estimate given in the scale's unit, resulted in 10.5 of a possible 45 points. MDC determines the smallest within-person change to ensure that the change is larger than the measurement error, and 10.5 points will provide an estimate of this limit when using the $\mathrm{BBQ}$ as an outcome measure. The results (Fig. 1) from LOA show that the large measurement error was equally spread across the whole scale range. This implies that when using the BBQ as an outcome to evaluate change during a treatment or clinical course, an observed change below 10.5 points can not be distinguished from measurement error, regardless of baseline value. This estimate of measurement error is large taking into consideration the scale from 0 to 45 . To the authors' knowledge, few studies have investigated the measurement error of the BBQ. Alamrani and coworkers obtained a somewhat lower MDC (5.9) and SEM (2.1) values influenced by their high ICC value (0.88) [15]. Due to the high measurement error in this study, more reports should investigate of measurement errors for the $\mathrm{BBQ}$, which may increase our confidence in utilizing the questionnaire as an outcome measure.

A high internal consistency was found for $\mathrm{BBQ}$, even though the Cronbach's alpha coefficient is sensitive to the number of items in the scale, and questionnaires with fewer than 10 items can result in a value that is too low $[38,39]$. The results are consistent with other studies conducted on BBQ, although our values of 0.82 (test) and 0.80 (retest) are slightly higher than in most previous studies. Previous publications have demonstrated
Cronbach's alphas ranging from 0.70 to $0.80[5,10,14-$ $17,31]$ and reflects the homogeneity of each statement.

Construct validity is an important element of the validity of a questionnaire. As there were no comparable questionnaires for evaluating back beliefs in our prospective cohort, construct validity was assessed by testing predefined hypotheses about expected correlations to other measurements in our study. The hypotheses were based on existing literature on the BBQ and its assumed relation to similar or non-similar constructs. As expected, a good correlation between the $\mathrm{BBQ}$ and the FABQ-PA was found, similar to the original study by Symonds et al., in Britain [5]. Other studies have been conducted on the BBQ and FABQ-PA with populations from different cultures and backgrounds, including Arabic and Chinese low back pain patients [14, 34] and Chinese healthcare students and workers [16, 17]. Their correlation analyses differ from ours and demonstrates low values ranging from -0.02 [16] to -0.35 [34]. These results show that healthcare professions and cultural background and origin are important aspects to consider when evaluating back beliefs and fear avoidance behavior due to physical activity. The moderate correlation between the BBQ and RMDQ was also as hypothesized, while the moderate correlation between the BBQ and PCS was slightly lower than expected. Two other studies have investigated the relationship between the Oswestry Disability Index and back beliefs and have found correlation between high disability status and negative back beliefs $[33,40]$. To the authors' knowledge, no previous studies have assessed the relationship between pain catastrophizing (PCS) and back beliefs (BBQ), making this study the first to investigate the correlation between these two scales. The divergent validity was shown by the low correlation found between the BBQ and NRS, and our results are consistent with previous research. Other studies investigating the relationship between the $\mathrm{BBQ}$ and pain are finding a low correlation, demonstrating that the degree of pain experienced is not related to pessimistic back beliefs $[15,17,18,33]$. The exception is that patients with a 1-week history of severe back pain might have more pessimistic back beliefs [31]. The correlation analysis confirmed 75\% of the predefined hypotheses, indicating a good construct validity [19].

One limitation of this study is that we could not prohibit participants from seeking medical advice or treatment between recruitment, baseline testing and retesting, and their back beliefs may therefore have been influenced by healthcare or alternative care practitioners. Furthermore, participants were recruited in primary care, and due to practical and economic considerations, there are no data on potential study participants that declined to participate. 


\section{Conclusions}

This study indicates that the Norwegian version of the BBQ had acceptable test-retest reliability, internal consistency and construct validity when used on elderly patients in primary care with a new episode of back pain. Further investigations on the importance and consequences of back beliefs are recommended. Research should also explore how to influence irrational attitudes and beliefs about back pain.

\section{Supplementary information}

Supplementary information accompanies this paper at https://doi.org/10. 1186/s12891-019-2910-8.

Additional file 1. The Norwegian version of the Back Beliefs Questionnaire.

\section{Abbreviations}

BACE: BACk Complaints in Elders; BBQ: Back Beliefs Questionnaire; Cl: Confidence interval; FABQ-PA: Fear-Avoidance Beliefs Questionnaire for physical activity; ICC: Intraclass correlation coefficient; LOA: Limits of agreement; MDC: Minimal detectable change; NRS: Numeric Rating Scale; PCS: Pain Catastrophizing Scale; RMDQ: Roland Morris Disability Questionnaire; SD: Standard deviation; SEM: Standard error of measurement; T0: Baseline; T1: Retest

\section{Acknowledgements}

The authors thank the team in the BACE-N project for data collection and support.

\section{Authors' contributions}

MG was involved in the design and project management of the study, her role as principle investigator and project manager of the BACE-N. ØV and RM took part of data collection and preparation of the datasets. BL, KS, RM and AT designed the study and contributed to the statistical analysis and interpretation of results. AT drafted the manuscript and the revisions from the coauthors. All authors contributed in preparation of the article and all authors approved the final manuscript.

\section{Funding}

Funding for this study was provided by the Norwegian Fund for Postgraduate Education in Physiotherapy. The funding agency had no role in the study design, data collection and analysis, decision to publish or writing of the manuscript.

\section{Availability of data and materials}

The datasets generated and analyzed during the study are available from the corresponding author on reasonable request.

\section{Ethics approval and consent to participate}

The Regional Committees for Medical and Health Research Ethics approved the study plan (Reference number: 2014/1634). All participants signed a written informed consent prior to inclusion in the study.

\section{Consent for publication}

Not applicable.

\section{Competing interests}

The authors declare that they have no competing interests.

\section{Author details}

'Oslo Metropolitan University, Pilestredet 44, 0167 Oslo, Norway. ${ }^{2}$ Research and Communication Unit for Musculoskeletal Health, Oslo University Hospital, P.B. 4950, Nydalen, 0424 Oslo, Norway. ${ }^{3}$ Sunnaas HF, Bjørnemyrveien 11, 1453 Bjørnemyr, Norway.
Received: 1 March 2019 Accepted: 23 October 2019

Published online: 03 November 2019

\section{References}

1. GBD. Global, regional, and national disability-adjusted life-years (DALYs) for 359 diseases and injuries and healthy life expectancy (HALE) for 195 countries and territories, 1990-2017: a systematic analysis for the global burden of disease study 2017. Lancet (London, England). 2018;392(10159): 1859-922.

2. Freburger JK, Holmes GM, Agans RP, Jackman AM, Darter JD, Wallace AS, Castel LD, Kalsbeek WD, Carey TS. The rising prevalence of chronic low Back pain. Arch Intern Med. 2009;169(3):251-8.

3. Hartvigsen J, Hancock MJ, Kongsted A, Louw Q, Ferreira ML, Genevay S, Hoy D, Karppinen J, Pransky G, Sieper J, et al. What low back pain is and why we need to pay attention. Lancet (London, England). 2018:391(10137):2356-67.

4. Paeck T, Ferreira ML, Sun C, Lin CWC, Tiedemann A, Maher CG. Are older adults missing from low Back pain clinical trials? A systematic review and meta-analysis. Arthritis Care Res. 2014;66(8):1220-6.

5. Symonds TL, Burton AK, Tillotson KM, Main CJ. Do attitudes and beliefs influence work loss due to low back trouble? Occup Med(Lond). 1996;46(1):25-32.

6. Waddell G, Newton M, Henderson I, Somerville D, Main CJ. A fear-avoidance beliefs questionnaire (FABQ) and the role of fear-avoidance beliefs in chronic low back pain and disability. Pain. 1993:52(2):157-68.

7. Grotle M, Brox J, Glomsrød B, Lønn J, Veierød M, Vølllestad N. Clinical course and prognostic factors in acute low back pain: patients seeking help for their first time. Ann Rheum Dis. 2004;63:91-2.

8. George SZ, Beneciuk JM. Psychological predictors of recovery from low back pain: a prospective study. BMC Musculoskelet Disord. 2015;16(1):49.

9. Elfering A, Anne FM, Nicola J, Oezguer T, Urs M. Beliefs about Back pain predict the recovery rate over 52 consecutive weeks. Scand J Work Environ Health. 2009;35(6):437-45.

10. Elfering A, Müller U, Rolli Salathé C, Tamcan Ö, Mannion AF. Pessimistic back beliefs and lack of exercise: a longitudinal risk study in relation to shoulder, neck, and back pain. Psychol Health Med. 2015;20(7):767-80.

11. Buchbinder R, Jolley D, Wyatt M. Population based intervention to change back pain beliefs and disability: three part evaluation. BMJ. 2001;322(7301):1516.

12. Bowey-Morris J, Davis S, Purcell-Jones G, Watson PJ. Beliefs about Back pain: results of a population survey of working age adults. Clin J Pain. 2011;27(3): 214-24.

13. Werner EL, Ihlebæk C, Lærum E, Wormgoor MEA, Indahl A. Low back pain media campaign: no effect on sickness behaviour. Patient Educ Couns. 2008:71(2):198-203.

14. Maki D, Rajab E, Watson PJ, Critchley DJ. Cross-cultural translation, adaptation and psychometric properties of the back beliefs questionnaire in modern standard Arabic, vol. 101; 2015. p. e937.

15. Alamrani $\mathrm{S}$, Alsobayel H, Alnahdi AH, Moloney N, Mackey M: Cross-Cultura Adaptation and Validation of the Back Beliefs Questionnaire to the Arabic Language. Spine 2016;41(11):E681-68.

16. Burnett A, Sze CC, Tam SM, Yeung KM, Leong M, Wang WT, Tan BK, O'Sullivan P. A cross-cultural study of the Back pain beliefs of female undergraduate healthcare students. Clin J Pain. 2009;25(1):20.

17. Chen G, Tan BK, Jia H-L, O'Sullivan P, Burnett A. Questionnaires to examine Back pain beliefs held by health care professionals: a psychometric evaluation of simplified Chinese versions. Spine. 2011;36(18):1505.

18. Dupeyron A, Lanhers C, Bastide S, Alonso S, Toulotte M, Jourdan C, Coudeyre E. The Back Belief Questionnaire is efficient to assess false beliefs and related fear in low back pain populations: A transcultural adaptation and validation study.(Research Article). PLOS ONE. 2017;12(12):e0186753.

19. Terwee CB, Bot SDM, de Boer MR, van der Windt DAWM, Knol DL, Dekker J, Bouter LM, de Vet HCW. Quality criteria were proposed for measurement properties of health status questionnaires. J Clin Epidemiol. 2007;60(1):34-42.

20. Scheele J, Luijsterburg P, Ferreira ML, Maher CG, Pereira L, Peul WC, van Tulder MW, Bohnen AM, Berger MY, Bierma-Zeinstra SMA, et al. Back Complaints in the Elders (BACE); design of cohort studies in primary care: an international consortium.(Study protocol)(Report). BMC Musculoskelet Disord. 2011:12:193.

21. Beaton DE, Bombardier C, Guillemin F, Ferraz MB. Guidelines for the process of cross-cultural adaptation of self-report measures. Spine. 2000; 25(24):3186-91. 
22. Roland M, Morris R. A study of the natural history of back pain. Part I: development of a reliable and sensitive measure of disability in low-back pain. Spine (Phila Pa 1976). 1983;8(2):141-4.

23. Sullivan MJL, Bishop SR, Pivik J, Butcher JN. The pain Catastrophizing scale: development and validation. Psychol Assess. 1995;7(4):524-32.

24. Grotle M, Vøllestad NK, Brox Jl. Reliability, validity and responsiveness of the fear-avoidance beliefs questionaire: methodological aspects of the Norwegian version. J Rehabil Med. 2006;38(6):346-53.

25. Grotle M, Vøllestad M, Brox M. Cross-cultural adaptation of the Norwegian versions of the Roland-Morris disability questionnaire and the Oswestry disability index. J Rehabil Med. 2003;35(5):241-7.

26. Fernandes L, Storheim K, Lochting I, Grotle M. Cross-cultural adaptation and validation of the Norwegian pain catastrophizing scale in patients with low back pain; 2012

27. Strong J, Ashton R, Chant D. Pain intensity measurement in chronic low back pain. Clin J Pain. 1991;7(3):209-18.

28. Bland JM, Altman DG. Statistical methods for assessing agreement between two methods of clinical measurement. Lancet. 1986;1 (8476):307-10.

29. Andresen EM. Criteria for assessing the tools of disability outcomes research. Arch Phys Med Rehabil. 2000;81:S15-20.

30. Cohen J. Statistical power analysis for the behavioral sciences. Hillsdale: Lawrence Earlbaum Associates; 1988.

31. Bostick GP, Schopflocher D, Gross DP. Validity evidence for the back beliefs questionnaire in the general population. Eur J Pain. 2013;17(7):1074-81.

32. Hoy D, Bain C, Williams G, March L, Brooks P, Blyth F, Woolf A, Vos T, Buchbinder R. A systematic review of the global prevalence of low back pain. Arthritis Rheum. 2012;64(6):2028-37.

33. Urquhart DM, Bell RJ, Cicuttini FM, Cui J, Forbes A, Davis SR. Negative beliefs about low back pain are associated with high pain intensity and high level disability in community-based women.(Research article)(Report). BMC Musculoskel Disord. 2008;9(148):148

34. Cheung P, Wong C, Cheung J. Psychometric validation of the crossculturally adapted traditional Chinese version of the Back beliefs questionnaire (BBQ) and fear-avoidance beliefs questionnaire (FABQ). Eur Spine J. 2018:27(8):1724-33.

35. Kaye AD, Baluch A, Scott JT. Pain management in the elderly population: a review. Ochsner J. 2010;10(3):179.

36. Dionne CE, Dunn KM, Croft PR. Does back pain prevalence really decrease with increasing age? A systematic review. Age Ageing. 2006:35(3):229-34.

37. De Vet HC, Terwee CB, Mokkink LB, Knol DL. Measurement in medicine: a practical guide. Cambridge: Cambridge University Press; 2011.

38. Pallant J. SPSS survival manual : a step by step guide to data analysis using IBM SPSS. 6th ed. Maidenhead: McGraw Hill; 2016.

39. Tavakol M, Dennick R. Making sense of Cronbach's alpha. Int J Med Educ. 2011:2:53-5.

40. Briggs AM, Jordan JE, Buchbinder R, Burnett AF, O'sullivan PB, Chua JYY, Osborne RH, Straker LM. Health literacy and beliefs among a community cohort with and without chronic low back pain. Pain. 2010;150(2):275-83.

\section{Publisher's Note}

Springer Nature remains neutral with regard to jurisdictional claims in published maps and institutional affiliations.

Ready to submit your research? Choose BMC and benefit from:
- fast, convenient online submission
- thorough peer review by experienced researchers in your field
- rapid publication on acceptance
- support for research data, including large and complex data types
- gold Open Access which fosters wider collaboration and increased citations
- maximum visibility for your research: over 100M website views per year
At BMC, research is always in progress.
Learn more biomedcentral.com/submissions

